\title{
The human capacity to transmit olfactory information*
}

\author{
J. A. DESOR† and GARY K. BEAUCHAMP†† \\ Monell Chemical Senses Center and Department of Otorhinolaryngology and Human Communication \\ University of Pennsylvania, Philadelphia, Pennsylvania 19104
}

\begin{abstract}
Previous estimates of the capacity of the olfactory channel are uniformly low. Either olfaction is considerably more limited than vision and audition or its capacity has been underestimated. It was suggested that underestimation may be due to use of stimuli having low levels of information or lack of laboratory training. Here, whole odors from objects were used. Naive Ss performed at levels consistent with earlier reports, and evidenced effects of prior experience in identifying the odors. With training, a much greater capacity than found previously was observed. It appears that the odors of single compounds and objects differ in information, dimensionality, or patterning, much like color patches and pictures or tones and words differ.
\end{abstract}

Channel capacity, the amount of information a sensory channel is able to carry, has been measured for olfaction. The estimates are fairly consistent and uniformly low. Engen and Pfaffmann (1959, 1960), using the odors of single compounds, found that adults were able to make approximately 4 correct absolute identifications of odors differing only in intensity and approximately 16 of odors differing in quality. That is, the olfactory system obtained sufficient information from singly presented stimuli to correctly classify them into 1 of 4 intensity categories or 1 of 16 quality categories. In information theory terms, the Ss were able to make two dichotomous decisions (use 2 bits of information) in identifying which intensity level was present or four dichotomies (use 4 bits of information) in identifying which odor quality was being perceived. Jones (1968) observed similar performance levels: after extensive laboratory training, one $S$ was able to make up to 24 correct absolute identifications, implying a channel capacity of 4.6 bits of information.

In trying to find any one $S$ who could demonstrate use of more olfactory information, Jones (1968) tested a chemist and two perfumers with odors selected to take advantage of their extensive olfactory experience. The chemist correctly identified 16 of 45 odors, a level consistent with Engen and Pfaffmann's (1960) estimate. Jones (1968) reported that the perfumers correctly recognized 150 and 164 of 192 odors presented. He presented these odors in 12 series of 16 and had the perfumers eliminate each odor from a list

*This research was supported in part by NIH Postdoctoral Fellowship 1 F02 NS50797-01 awarded to J.A.D. and NIMH Postdoctoral Fellowship 1 F02 MH53301-01 awarded to G.K.B.

†Requests for reprints should be sent to J. A. Desor, Monell Chemical Senses Center, University of Pennsylvania, 3500 Market Street, Philadelphia, Pennsylvania 19104.

††We express our appreciation to Mark Gromko and John Rogers for their invaluable assistance. of the 16 correct responses given with each series. Thus, his testing method required only repeated use of 4 bits of information to differentiate each odor from the 15 alternatives presented in the series with it. The perfumers correctly matched an average of 13.1 of 16 stimuli with their names, which would have required 3.7 bits of information had they been absolute identifications.

Two additional estimates of olfactory channel capacity are available from studies directed at other issues. In both cases, naive Ss were presented with stimuli that were or included odors of common objects or materials and were asked to name the odorous item. These stimuli, mediating perception of objects or materials, are likely to be highly patterned. Mozell, Smith, Smith, Sullivan, and Swender (1969) delivered 20 stimuli orally while their odors were given in a nasal airstream. The mean number of correct identifications of the 17 clearly odorous stimuli, calculated from graphically presented data, was 10.1, a level suggesting use of 3.4 bits of sensory information. Engen and Ross (1973) presented 20 relatively common odors for absolute identification. They found that naive Ss could identify or give "good associations" for an average of 10.9 of them, performance requiring use of 3.3 bits of information per stimulus.

The above estimates, taken from several studies using a variety of stimuli and procedures, suggest that humans can correctly classify the odors they encounter into somewhere between 10 and 24 categories. That is, they appear to be able to identify between 10 and 24 odors in an absolute way, or use between 3.3 and 4.6 bits of olfactory information. This is far below the levels assumed to exist in the course of normal perception and identification where it is clear that very large numbers of stimuli (e.g., faces, words) are identified absolutely, i.e., without direct comparison with every possible alternative. 
One possibility is that olfaction is considerably more limited than vision and audition, and that large numbers of odors, even highly patterned ones, are not identiliable in an absolute way. There is some evidence for this in addition to the uniformly low performance of olfaction described above. After measuring channel capacity for qualitatively different odors. Engen and Pfaftmann (1960) tested for the predicted improvement in performance when the intensity dimension was added to these. The presumed increase in number of stimulus dimensions did not alter performance; Ss continued to exhibit use of approximately 4 bits of information although a greater amount of sensory information had been presented to the channel. This suggests that the observed capacity of 4 bits reflects an overall limit in the channel rather than merely use of stimuli having only low levels of information.

The alternative to concluding that a major sensory system is restricted to dealing with stimuli at the level of color patches or tones is to assume that previous studies have not measured the maximum capability of olfaction. Among the variables that may have suppressed maximal performance in the previous studies are (a) use of stimuli having only low levels of information (analogous to selecting color patches or tones rather than objects or words for stimuli) and (b) lack of training with odors and/or the verbal labelling of them.

It is the purpose here to determine whether the previous studies have underestimated the capacity of the olfactory channel by using stimuli that are likely to offer high levels of information and giving Ss laboratory training with them. The first study was designed to test naive Ss' ability to make absolute identitications of the whole odors of common, odorous objects or materials. By asking the Ss to name the object or material being perceived, the task was assumed to be analogous to visual identification of common objects when the object is named. It was expected that naive Ss would perform at levels consistent with those found by Engen and Ross (1973) and Mozell et al (1969), who used similar conditions. If the channel capacity observed in this case is an underestimation because of lack of training with either the odors or their names, there should be evidence of it. Specitically, there should be correlations between indices of past experience with the stimuli and ability to identify them. Although Engen and Ross (1973) found that judged familiarity of odors did not affect odor recognition, this index of prior experience may correlate with the measure of learning used here.

The purpose of the second study was to determine whether or not laboratory training facilitates performance on this task. Engen and Pfaffmann (1960) found no effects of extensive laboratory training on the ability to identify qualitatively different odors, and their Ss demonstrated a channel capacity of 4.0 bits even after "hundreds of judgments over a period of weeks [Engen, 1970, p. 379]." It is possible, however, that their stimuli, the odors of single compounds for the most part, did not offer more than 4 bits of information to a channel capable of carrying more than that. If the olfactory channel is more capable than now supposed, then training with objects, stimuli that offer high levels of information to other sensory systems, should lead to higher performance levels than previously observed.

\section{EXPERIMENT I}

\section{Method}

Subjects

The Ss were 15 female and 11 male adults who had had no prior experience with the procedure and no information as to the purpose of the study.

\section{Apparatus}

Stimuli. The stimuli were the odors emanating from 32 common odorous objects or materials. The items were placed in quart-size glass jars that had been coated with Teflon-lined tinfoil. The jar openings were first covered with a double layer of surgical gauze, then sealed with caps for at least 1 h prior to use. The 32 objects, listed on the abscissa of Fig. 1, were used in their most commonly encountered state (e.g., fresh green paper, fried liver; common, large-sclling brands of prepackaged itens). They were treated consistently for all presentations (e.g.. 35-50 grains of popcorn popped in 2 tsp of corn oil on day of testing), but no attempt was made to specify the compounds emanating from them or to insure that they were chemically constant. Thus, the defined stimuli were objects or materials. and their odors varied as much as those encountered outside the laboratory. One stimulus, designated "motor oil," was changed during the experiment. Household machinc oil was used for 9 Ss, and motor oil was used for the other 17 Ss.

Rating Scales. For each S, there was a packet of 32 slips, each with a confidence rating scale on it. There was a similar packet of 32 familiarity rating slips. Both rating scales were presented as five discrete categories ("very familiar" to "very unfamiliar" and "very sure is correct" to "very sure is wrong"), of which one was to be checked.

\section{Procedure}

The Ss were tested individually in a large, dimly lighted room with an air turnover rate specitied at $100 \% / \mathrm{h}$. The environment was intended to be normal olfactory space, and was considered as such if, in the E's judgment, there were no exceptionally strong or unusual odors.

The $S$ was seated across from the $E$ and given packets of familiarity and confidence rating slips. He was instructed as follows: "We're interested in finding out how well things are identified by their odors. I have 32 odor samples (indicating jars). l'll put each jar in front of you for you to smell. Don't touch then, just smell the odor coming from them. For each one, tell me what has that smell." Then he was told to indicate both how familiar each odor was to him and how sure he was that the answer he gave was correct by checking the appropriate alternatives on the rating seales.

The 32 stimuli were presented in a randomized order that varied across Ss. Each jar was unsealed (retaining its gauze cover), placed in front of the $S$, and left there for $30 \mathrm{sec}$. There were $10-15 \mathrm{sec}$ between presentations. The first noun given in identitication was recorded as the S'S response, and latency to this noun was measured. 


\section{Results \\ Correctness of Identifications}

The first noun given in identification was scored for correctness. The a priori scoring system did not anticipate all of the responses, and thus, a posteriori scoring was necessary. The responses to each stimulus were categorized into five levels of correctness, designated by scores of $5-1$ as follows:

5: Precisely correct-an exact designation of the stimulus at the level typically used to order it (e.g., "canned tomatoes" or "tomato" for canned tomatoes).

4: Nearly correct-an item with significant overlap of components with the stimulus (e.g., "tomato juice," "tomato soup," or "catsup" for canned tomatoes).

3: Somewhat correct-an item in the same narrow class as the stimulus (e.g., "wine" or "whiskey" for beer) or designation of that class (e.g., "alcohol" or "liquor" for beer).

2: Nearly incorrect-an item in the same medium-sized class as the stimulus (e.g., "beef" for tunafish, "urine" for cat feces) or designation of that class (e.g., "vegetable" for canned tomatoes).
1: Completely incorrect--an item not meeting any of the above criteria (e.g., "canvas cloth" or "meat" for crayons), including designation of a global class (e.g., "food" for canned tomatoes).

0 : No response given during the 30 -sec trial.

The number of precisely correct ( 5 above) identifications per $S$ ranged from 2 to $21(\mathrm{M}=12.3)$. If the nearly correct responses are included (4 and 5 above), Ss identified an average of 15.3 of the 32 stimuli (range 3-26). The means and ranges of the number of responses per $S$ given scores of $3,2,1$, and 0 were $2.9(0-6), 1.8(0-5), 7.8(1-17)$, and $4.2(0-24)$, respectively.

The stimuli differed in how often they were identified from their odors. Fresh ground coffee was called "coffee," "ground coffee," or "coffee grounds" by 22 of the $26 \mathrm{Ss}$, whereas 2 Ss called an unsmoked cigar (common brand) "cigar." The 32 stimuli and the number of Ss giving identifications for each at Correctness Levels 5 and 4 ("correct"), 3 and 2 ("intermediate"), and 1 ("incorrect") are given in Fig. 1. The number by which 26 exceeds the total of these is the number of Ss failing to give any identification of the stimulus.

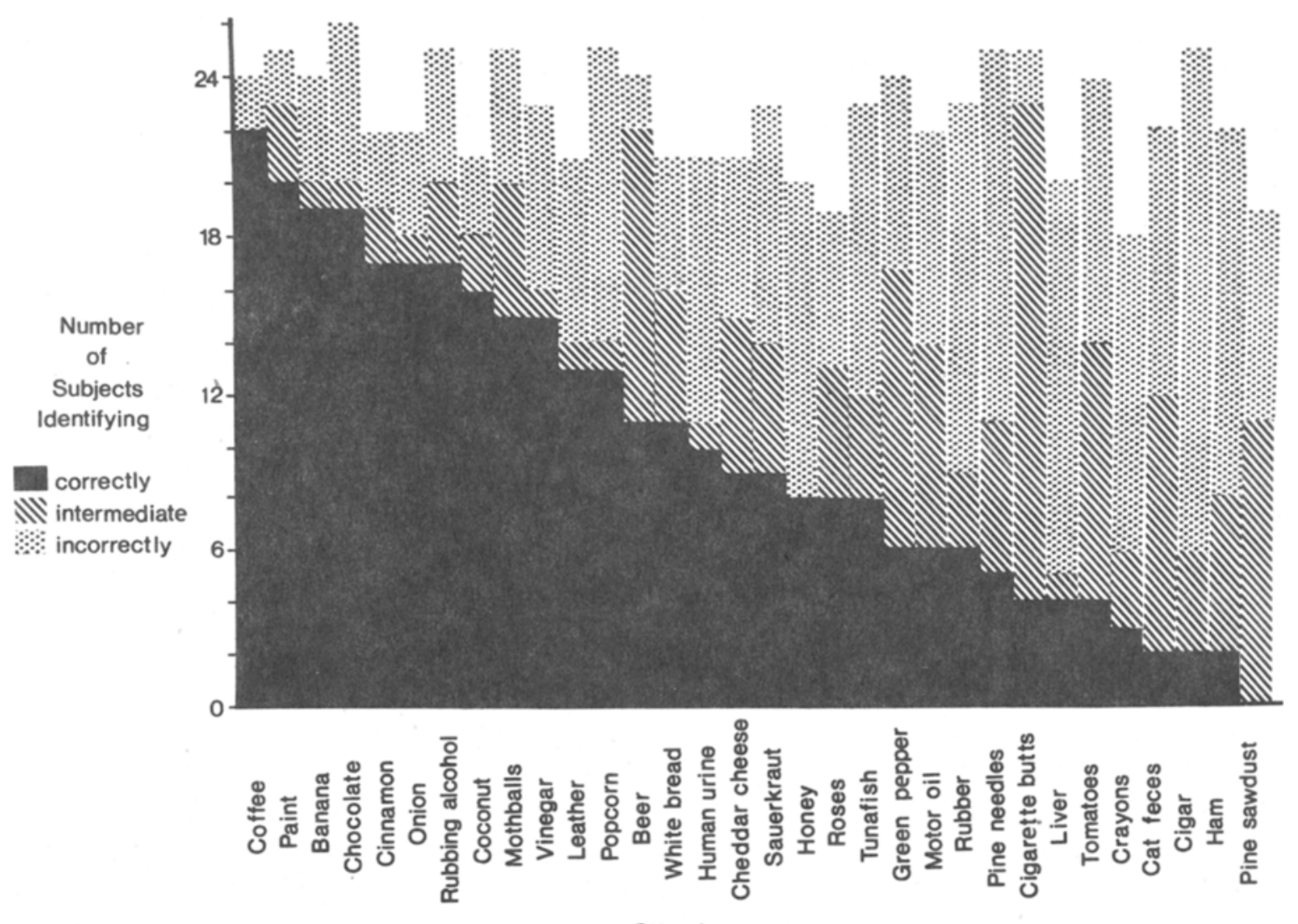

Stimulus

Fig. 1. Number of $S s(N=26)$ identifying each of 32 stimuli correctly (scores of 4 or 5 ), intermediately correct (score of 3 ), and incorrectly (scores of 2 or 1 ). 


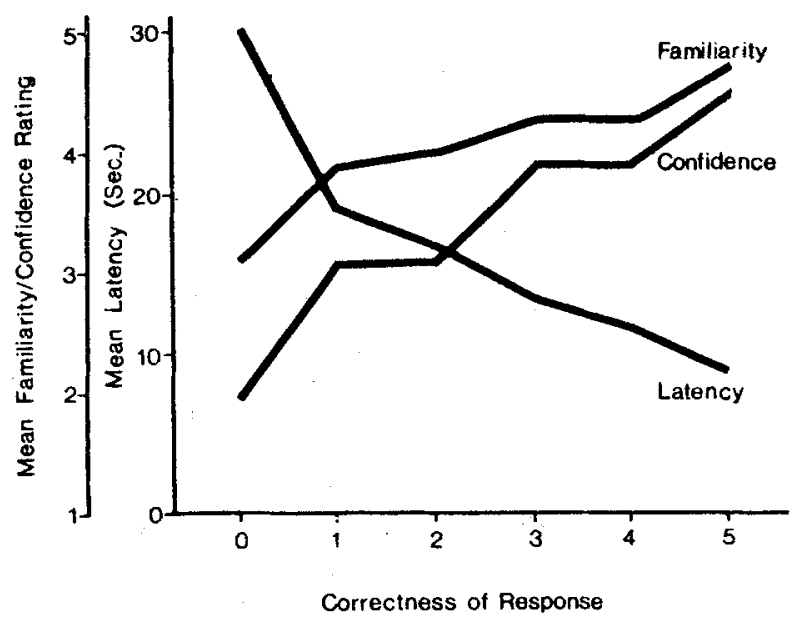

Fig. 2. Mean latency of response, familiarity with the stimulus, and confidence in the correctness of response as a function of the correctness of the identification given. Responses of $26 \mathrm{Ss}$ to 32 stimuli.

\section{Latency, Familiarity, and Confidence (Fig. 2)}

The latency of the Ss' responses correlated with their correctness $(\mathrm{r}=-.59$, $\mathrm{df}=831, \mathrm{p}<.001)$, with the more correct identifications being given in less time. Ss' ratings of how familiar the odors were correlated positively with the correctness of their identification $(\mathrm{r}=.48, \mathrm{df}=831, \mathrm{p}<.001)$. In addition, the Ss' ratings of how sure they were that their identifications were correct (confidence) correlated with the actual correctness $(\mathrm{r}=.65, \mathrm{df}=$ $831, \mathrm{p}<.001)$.

\section{Discussion}

The naive Ss making olfactory object identifications performed at levels similar to those found previously for olfactory stimuli. If precisely and nearly correct responses are included, the mean capacity was 3.9 bits and the maxinum was 4.7 bits. These levels are considerably lower than expected for the highly patterned stimuli that objects are assumed to offer.

There is evidence that this low performance was due to a lack of previous learning rather than limits inherent in the olfactory channel. Differences in each of four measures suggest that incorrect responding was due to lower levels of prior learning. Familiarity, a measure of prior experience with the stimuli, correlated with the correctness of identification. Measures of latency also suggested that the more correct responses were the better learned ones. Further, Ss' confidence in the correctness of their identifications correlated with actual correctness. That is, the Ss discriminated between their correct and incorrect labels. It appears that errors were made because Ss were unable to give the correct label rather than their being unable to discriminate the stimulus from the alternative named. In addition, the stimuli differed on how often they were correctly identified. If the low performance had been due only to confusion between undifferentiable stimuli, stimuli with low levels of information in them, one might expect a more equal distribution of errors among the confused alternatives.

\section{EXPERIMENT II}

If the remarkably poor ability in identifying odors reflects a lack of training in this task, as the above data suggest, then laboratory training should greatly increase performance levels. The purpose here is to test for the effects of training on measurements of olfactory channel capacity using objects as stimuli.

\section{Method}

Subjects

The Ss were onc temale and two male adults. All had participated in pilot work and thus were somewhat familiar with the stimuli.

\section{Apparatus}

The stimuli described in Experiment I were used for a set of 32 odors. For a set of 64 stimuli, 26 of the original 32 and 38 additional objects or materials were used. The 38 new stimuli included 24 foods and seasonings (e.g., molasses, dill pickle, soy sauce, celery seed), 10 nonfood items (e.g., musty book, ivy, pencil sharpener shavings), and 2 synthetic odors (sweat, lilac). All stimuli were detined and prepared as described in Experiment $\mathbf{l}$.

\section{Procedure}

The method of presenting stimuli was that used in Experiment I, except that the $S$ was told the identity of the object he was smelling immediately after he responded with his first noun, or, if he gave no response, after $30 \mathrm{sec}$.

The procedure was designed to train the $S s$ to maximal performance levels as quickly as possible. The Ss were told which stimuli were to be included in each set prior to the training sessions. The three Ss were given training with the set of 32 odors until a critcrion of two successive sessions in which no errors were made was reached. They were tested individually two or three times per day with at least $1 \mathrm{~h}$ between sessions. Each of the 32 stimuli was presented once per session in a randomized order. The first noun given in identitication and the latency to this noun were recorded. Five days after the last $S$ had reached cirterion. a group session was given in which each of the 32 stimuli was presented three times. The order of presentation was randomized for the entire 96 presentations to prevent the Ss from remembering and eliminating as a possibility an object that had already been presented. For group sessions, the $S s$ wrote their identifications, and all were given the correct response after all had responded or after $30 \mathrm{sec}$.

Forty days after the above, the three $S$ s were trained as a group with the set of 64 odors. The new 38 stimuli were divided into two sets of 20 and 18 . First, five sessions were given with the set of 20 , then two were given with the set of 18 . These sets were then combined, and three sessions were given with the 38 . Then, one session with the 26 stimuli from the original set of 32 and one session with the entire set of 64 were given. Each stimulus in the set being tested was present once per session during the above training. Four days after this training, a test session was given. Each of the 64 stimuli appeared three times during a single session, and the order of presentation was randomized over all 192. 


\section{Results}

In the course of training, Ss were tested with sets of $18,20,32,38$, and 64 stimuli. The mean numbers correctly identified in the last session given with each set are given in Fig. 3 ("whole odors-training"). Performance of the naive $S s$ in Experiment $I$ and previously reported levels are included for comparison.

All three Ss learned to identify the 32 objects by their odors alone, performance requiring 5 bits of olfactory information. The numbers of trials needed to reach criterion were 5,9 , and 11 for the three Ss. In the final session, with 96 presentations, the three Ss correctly identified 94,95 , and 96 of them. The mean response latency for those stimuli that were correctly identified on all trials by a $S$ decreased from $6.1 \mathrm{sec}$ on the first trial to $2.8 \mathrm{sec}$ on the criterion trial $(\mathrm{t}=$ 3.90, df $=74, \mathrm{p}<.001$ ).

The three Ss approached perfect performance when training was extended to 64 objects (Fig. 3); in the last session, they correctly identified 173,177 , and 182 of the 192 stimuli presented. These performance levels required use of $5.85,5.88$, and 5.92 bits of information, respectively.

\section{Discussion}

With laboratory training, the Ss reached perfect performance in identifying absolutely the set of 32 stimuli used in Experiment I. Whereas the naive Ss demonstrated use of 3.9 bits of olfactory information, a capacity of 5 bits was reached with practice. In contrast to Engen's (1970) report, there was clear evidence that identifying qualitatively different odors is affected by training. Both the number of errors and the latency to correct responses decreased with practice.

When the task was increased to one requiring 6 bits of information for perfect performance, the Ss demonstrated use of approximately 5.9 bits. This capacity was observed on a test trial following some pretraining and only one practice trial, and thus may not be the maximum possible.

\section{CONCLUSIONS}

The capacity of the olfactory channel was found to be considerably higher than previously supposed. It is capable of carrying at least 5.9 bits of sensory information, which is equivalent to being able to classify singly presented odors into 1 of at least 60 categories. The earlier conclusion, that olfaction is limited to performing at levels similar to those found for colors or tones (Engen, 1970), underestimated the capacity of this sensory system.

Two factors, lack of training and use of stimuli having low levels of information, appear to account for the earlier underestimations. Naive Ss naming

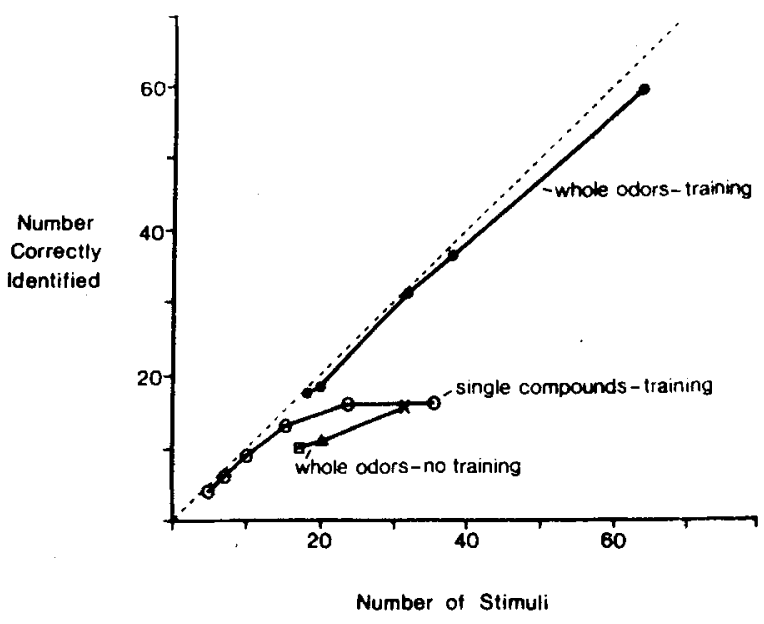

Fig. 3. Number of correct absolute identifications as a function of the number of stimuli presented. Performance is given for single compounds and whole odors (of objects or materials) after laboratory training and for whole odors without laboratory training. Data from Experiment I (X), Experiment II $(\bullet)$, Engen and Pfaffmann, $1960(O)$, Mozell et al, $1969(\square)$, and Engen and Ross, $1973(\triangle)$.

odorous objects or materials performed here at levels similar to those found by previous investigators under similar conditions. The correctness of their identifications, however, correlated with latency, confidence ratings, and judged familiarity with the stimulus. This strongly suggested that errors could be eliminated with training and, thus, did not reflect an inability to process greater amounts of olfactory information. Training did facilitate performance. The naive Ss gave precisely or nearly correct identifications for an average of 15.3 stimuli, whereas after 5 to 11 trials, the three trained Ss were able to identify the entire set of 32 stimuli. Presumably the naive Ss tested previously with common odors (Mozell et al, 1969; Engen \& Ross, 1973) would have performed at higher levels after practice.

Lack of training is not a tenable explanation for Engen and Pfaffmann's (1960) low estimate, since their Ss received considerably more training than was given here with no effect. Their procedure involved the learning of correct labels as well as extensive practice in making absolute identifications of odors, and performance was consistent at 4 bits (use of 16 categories). Since the results here make it clear that the channel is not limited to carrying 4 bits of information, it is reasonable to assume that the other limiting factor, the amount of information offered to the channel, was operating in their studies.

All except one of their stimuli were the odors of single compounds, whereas, here, the stimuli were the whole odors emanating from objects or materials (e.g., honey, cinnamon). It is likely that olfactory stimuli differ in level of information just as color 
patches and tones offer less information than the highly patterned stimuli involved in full perception (e.g., faces, objects, words, melodies). Although currently there is no adequate classification system for odors, it might be expected that objects offer highly patterned olfactory stimuli. That $S$ s responded to Engen and Pfaffmann's (1960) stimuli as if they were two- or three-dimensional (Miller, 1956) suggests that this set of odors are at the level of color patches or tones.

\section{REFERENCES}

ENGEN, T. Man's ability to perceive odors. In J. Johnston, D. Moulton, and A. Turk (Eds.), Advances in chemoreception. Vol. 1. Communication by chemical signals. New York: Appleton-Century-Crofts, 1970.
Engen, T., \& Pfaffmann, C. Absolute judgments of odor intensity. Journal of Experimental Psychology, 1959, 58, 23-26.

Engen, T., \& Pfaffmann, C. Absolute judgment of odor quality. Journal of Experimental Psychology, 1960, 59, 214-219.

Engen, T., \& Ross, B. Long-term memory of odors with and without verbal descriptions. Journal of Experimental Psychology, 1973, 100, 221-227.

Jones, F. Information content of olfactory quality. In N. Tanyolac (Ed.). Theories of odor and odor measurement. Bebek, Istanbul: Robert College Research Center, 1968.

Miller, G. The magical number seven, plus or minus two: Some limits on our capacity for processing information. Psychological Review, 1956, 63, 81-97.

Mozell, M.. Smith, B., Smith, P., Sullivan, R., \& Swender, P. Nasal chemoreception in flavor identification. Archives of Otolaryngology, 1969, 90, 367-373.

(Received for publication May 19, 1974; revision accepted July 18,1974 .) 